\title{
Self-Delayed Feedback Based Car Following Control With Velocity Uncertainty of Preceding Vehicle On Gradient Roads
}

Cong Zhai ( $\square 957083516 @ q q . c o m)$

South China Normal University

\section{Weitiao Wu}

South China University of Technology https://orcid.org/0000-0001-9047-0812

\section{Research Article}

Keywords: Car following model, Self-delayed feedback controller, Gradient road, Frequency domain, Stability

Posted Date: June 17th, 2021

DOI: https://doi.org/10.21203/rs.3.rs-513299/v1

License: (c) (i) This work is licensed under a Creative Commons Attribution 4.0 International License.

Read Full License

Version of Record: A version of this preprint was published at Nonlinear Dynamics on October 21st, 2021. See the published version at https://doi.org/10.1007/s11071-021-06970-7. 


\title{
Self-delayed feedback based car following control with velocity uncertainty of preceding vehicle on gradient roads
}

\author{
Cong Zhai ${ }^{1,2}$; Weitiao $\mathrm{Wu}^{1 *}$ \\ (1. School of Civil Engineering and Transportation, South China University of \\ Technology, Guangzhou 510641 Guangdong China \\ 2. School of Transportation and Civil Engineering and Architecture, Foshan University, Foshan \\ 528000 Guangdong China)
}

\begin{abstract}
Uphill and downhill roads are prevalent in mountainous areas and freeways. Despite the advancement of vehicle-to-vehicle (V2V) communication technology, the driving field of vision is still largely limited under such a complex road environment, which hinders the sensors accurately perceiving the speed of the front vehicle. As such, a fundamental question for autonomous traffic management is how to control traffic flow associated with the velocity uncertainty of preceding vehicles? This paper seeks to develop a controlling framework for corporative car following control under such complex road environment. To this end, we first propose a traffic flow model accounting for the uncertainty effect of preceding vehicles velocity on gradient road. We further design a new self-delayed feedback controller based on the velocity and headway difference between the current time step and historical time step, in an aim to enhance the robustness of traffic flow. The sufficient condition where traffic jam does not occur is derived from the perspective of the frequency domain via Hurwitz criteria and $H_{\infty}$ norm of transfer functions. The bode diagram reveals that the robustness of closed-loop traffic flow model has been significantly enhanced. We also conduct simulations to verify the theoretical analysis.
\end{abstract}

Keywords: Car following model; Self-delayed feedback controller; Gradient road; Frequency domain; Stability

\section{Introduction}

Over the past decades, the rapid development of urban economy has spurred the growth of car ownership and traffic congestion. Along with this, a variety of approaches and countermeasures have been proposed to alleviate traffic congestion by making use of emerging technology. On one hand, numerous traffic management and planning approaches have emerged, such as bus route optimization tactics [1-2] and online electric vehicle charge scheduling [3]. On the other hand, another stream of research direction mainly concentrated on the development of traffic flow models to reproduce traffic congestion and explore the underlying mechanism. Motivated by the advancement in V2V communication technology, this paper devises a self-delayed feedback control for autonomous traffic considering uncertain velocity of preceding vehicle on gradient road. The autonomous traffic is modeled as a car-following model with a designed controller.

According to the research subjects, current traffic flow theoretical models can generally be divided into following two branches: macroscopic and microscopic traffic flow models. The former mainly includes the continuous models [4-8] and lattice hydrodynamic models [9-15], while the latter includes the car-following models [16-18] and the cellular automata models

Corresponding Author: ctwtwu@scut.edu.cn 
[19-20]. In the former category, a number of en-route vehicles are modeled as compressible continuous fluid with the time- and location-dependent vehicle density and average speed. By constructing the partial differential equations of local density and local velocity, the dynamic behavior of traffic flow can be revealed by solving the equations. Although such type of models is less computationally expensive, it cannot analyze the microscopic driving behavior of the vehicle. In contrast, the latter takes individual vehicles as the research subject, and establishes differential equations based on the speed, headway and acceleration information of vehicles to study the vehicle motion behavior. In 1995, a seminal work of car-following model were put forward by Bando, termed optimal velocity $(\mathrm{OV})$ model $^{[21]}$. The optimal velocity function has an inflection point and elegant mathematical and physical properties, which facilitates linear and nonlinear theoretical derivation. Since then, the OV model has attracted wide attentions, yielding a number of extended and more realistic traffic flow models. The representative works can be referred to Table 1.

Table 1 An summary of OV model research in recent years

\begin{tabular}{ccc}
\hline Author & Characteristics & Remark \\
\hline Jiao & Collision sensitivity & literature [22] \\
Yu, Zhao & Acceleration & literatures [23-24] \\
Xu & Asymmetric optimal velocity & literature [25] \\
Sun, Jin, Sun & Average speed & literatures [26-28] \\
Ma, Ma, Chen & Backward looking & literatures [29-32] \\
Li, Sun, Li, Yan & Electronic throttle angle & literatures [33-36] \\
Hou, Zhou, Zheng & Visual angle & literatures [37-39] \\
Li, Li, Jin, Li & Non-lane-discipline & literatures [40-43] \\
Tang & Driver's bounded rationality & literatures [44-45] \\
Zhai, Song, Liu & Traffic jerk & literatures [46-48] \\
Tang, Zhang & Traffic interruption probability & literatures [49-50] \\
Li, Kuang & Multiple effect & literatures [51-52] \\
Tang, Kuang & Honk effect & literatures [53-54] \\
Zhai, Sun & Road condition & literatures [55-56] \\
Cao, Yu, Wang & Memory effect & literatures [57-59] \\
Tang, Wang, Zhou & Anticipation effect & literatures [60-62] \\
Yu, Li & Delay effect & literatures [63-64] \\
Peng, Tang & Driver's characteristic & literatures [65-66] \\
Wang, Sun & Driver's desire & literatures [67-68] \\
\hline
\end{tabular}

In practice, the gradient roads are not uncommon in the economically underdeveloped areas or mountainous freeways, as shown in Fig.1. The road slope may exert great influence on road traffic jam [69-70]. Despite the development of Internet of Vehicles, the driving field of vision is still limited in a traffic environment with gradient roads, which hinders the sensors perceiving the speed of the front vehicle accurately. Therefore, the velocity uncertainty of preceding vehicles should not be neglected in such complex road environment. However, to date, the existing studies on traffic flow assume that the current vehicle can accurately receive the headway or velocity information of preceding vehicles. Motivated by this fact, there is an increasing need to devise systematic traffic flow control algorithm for such traffic environment. To this end, we first propose an extended traffic flow model accounting for the effect of velocity uncertainty of preceding vehicle on the gradient road. It proceeds to design a new self-delayed feedback controller without relying on any extra traffic information. The stability criterion of closed-loop system is also derived. 
The structure of this paper is as follows: In Section 2, a modified car following model taking into account the effect of velocity uncertainty of preceding vehicle on gradient road is presented. In Section 3, the stability condition of closed-loop car following model is obtained via Hurwitz criterion and $H_{\infty}$ norm of transfer functions. In Section 4, some numerical examples are conducted out to verify the conclusions of the above theoretical analysis, finally, the conclusions and follow-up research works of this paper are given in Section 5.

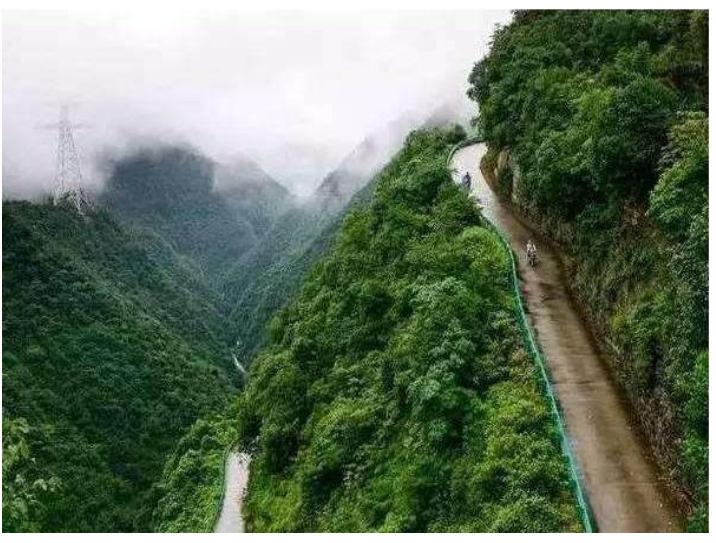

(a)

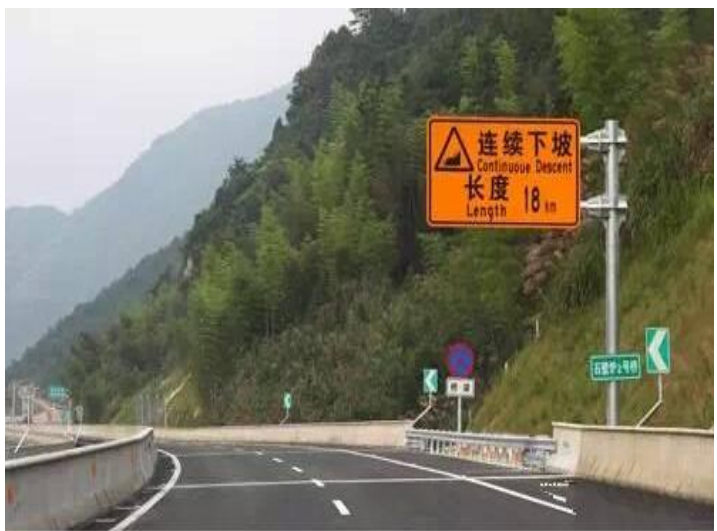

(c)

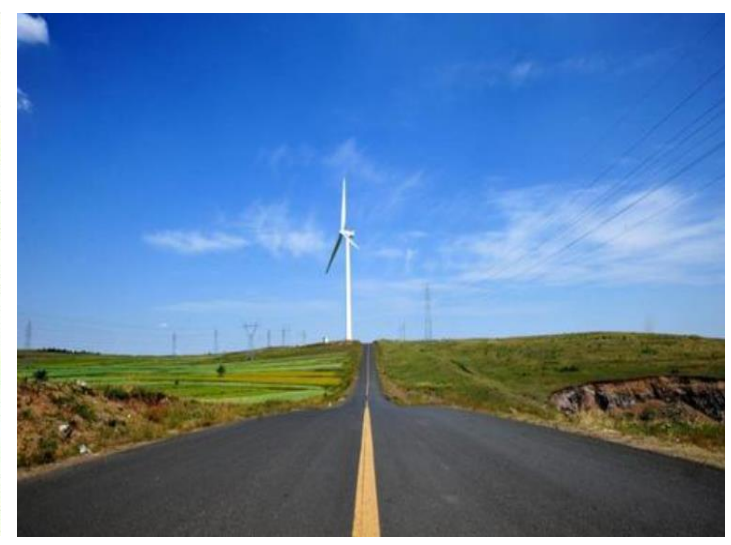

(b)

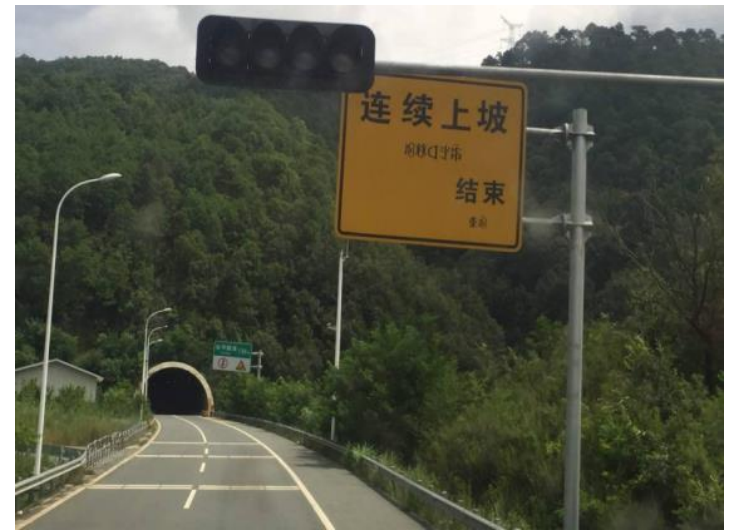

(d)

Fig.1 Common gradient road in urban and mountain area, (a) Hebei $66^{\text {th }}$ highway; (b) the $318^{\text {th }}$ highway in Guizhou Province; (c) Continuous downhill sign along the highway; (d) Continuous uphill sign along the highway

\section{Model}

In 1995, Bando [21] proposed an OV model to study the car following characteristic of vehicles on a single lane where the overtaking is forbidden, and the dynamic equation is expressed as:

$$
\begin{gathered}
\frac{d v_{n}(t)}{d t}=a\left[V\left(y_{n}(t)\right)-v_{n}(t)\right] \\
\frac{d y_{n}(t)}{d t}=v_{n+1}(t)-v_{n}(t)
\end{gathered}
$$

where $v_{n}(t)$ and $v_{n+1}(t)$ respectively represent the instantaneous velocity information of current vehicle $n$ and preceding vehicle $n+1$ at time $t$. $a$ represents the sensitivity of the driver. $y_{n}(t)$ represents the instantaneous headway of the target vehicle, $V(\mathrm{~g})$ represents the optimal velocity function, which is uniquely determined by the parameter $y_{n}(t)$, specifically

$$
V(y)=\frac{v_{\max }}{2}\left[\tanh \left(y-y_{c}\right)+\tanh \left(y_{c}\right)\right]
$$


where $v_{\max }$ and $y_{c}$ respectively represent the maximum speed and safe distance.

In order to eliminate the phenomenon of the excessive acceleration and unreasonable deceleration in the traditional optimal velocity model, Helbing and Tilch [71] presented an improved generalized force (GF) model expressed as follows:

$$
\frac{d v_{n}(t)}{d t}=a\left[V\left(y_{n}(t)\right)-v_{n}(t)\right]+\lambda H\left(-\Delta v_{n}(t)\right) \Delta v_{n}(t)
$$

where $\Delta v_{n}(t)$ represents the velocity difference between current vehicle $n$ and preceding vehicle $n+1$, and $\Delta v_{n}(t)=v_{n+1}(t)-v_{n}(t) . \quad \lambda$ is the corresponding weight coefficient, $H(\mathrm{~g})$ represents the Heaviside function, and

$$
H(x)=\left\{\begin{array}{l}
0, x \leq 0 \\
1, x>0
\end{array}\right.
$$

Later, in order to overcome the low starting wave speed in above-mentioned generalized force model, Jiang et al. [72] presented a full velocity difference (FVD) model, which is written as follows:

$$
\frac{d v_{n}(t)}{d t}=a\left[V\left(y_{n}(t)\right)-v_{n}(t)\right]+\lambda \Delta v_{n}(t)
$$

Since the FVD model, extensive derivative models are developed. considering that uphill and downhill roads are prevalent in mountainous areas and freeways, the literature [69] proposed a traffic flow model considering road slope information to study the impact of road slope information on traffic jam, and the kinetic equation is as follows

$$
\frac{d v_{n}(t)}{d t}=a\left[V^{o p}\left(y_{n}(t)\right)-v_{n}(t)\right]+\lambda \Delta v_{n}(t)
$$

where $V^{o p}(\mathrm{~g})$ represents the modified optimal velocity function constrained by the slope information, and the function is taken as follows:

$$
V^{o p}(y)=\frac{v_{f, \max }-v_{g, \max }(\theta)}{2}\left[\tanh \left(y-y_{c}(\theta)\right)+\tanh \left(y_{c}(\theta)\right)\right]
$$

where $v_{g, \max }(\theta)$ and $y_{c}(\theta)$ respectively represent the speed adjustment and safety distance item affected by the acceleration of gravity. $v_{f, \max }$ is the maximum velocity without any slope. Fig. 2 shows a schematic diagram of the force of the vehicle on the uphill and downhill scenarios. The specific value of $v_{\max }(\theta)$ of can be determined by

$$
v_{\max }(\theta)=\frac{m g \sin \theta}{\mu}
$$

where $m$ and $g$ respectively represent the mass of the vehicle and the acceleration of gravity, i.e. $g=9.8 m / s^{2} . \mu$ is the friction coefficient, to simplify the analysis we set $\mu=m g$. Since the safety distance differs in the uphill $(\theta>0)$ and downhill $(\theta<0)$ scenarios [69], we have that

$$
y_{c}(\theta)=y_{c}(1-\eta \sin \theta)
$$

where $h_{c}$ is the safety distance of vehicles on the general road without any slope information, $\xi$ is constant, which is taken as $\xi=1$ to simplify the analysis. As a result, the corresponding optimal speed function can be rewritten as

$$
V^{o p}(\rho)=\frac{v_{f, \max }-\sin \theta}{2} V_{0}(\rho)
$$

where 


$$
V_{0}(\rho)=\tanh \left(y-y_{c}(\theta)\right)+\tanh \left(y_{c}(\theta)\right)
$$

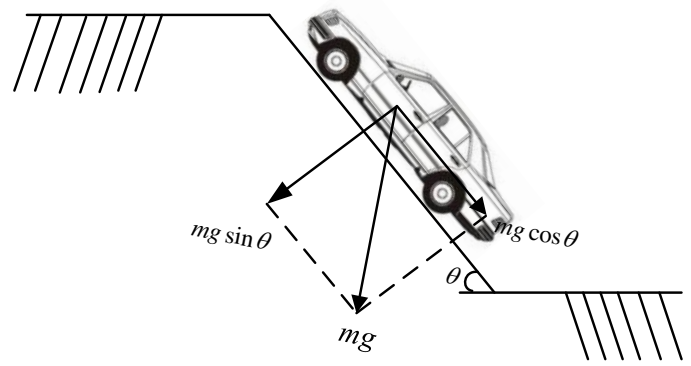

(a)

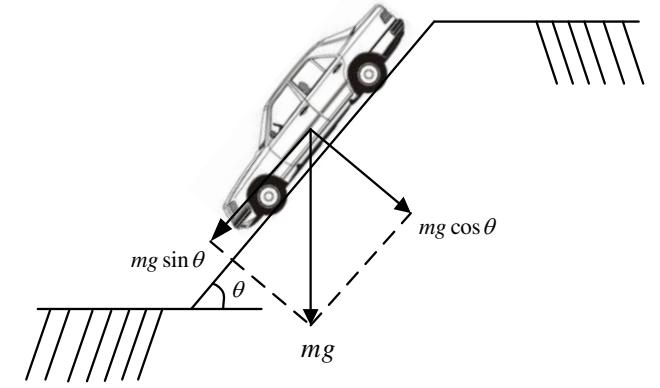

(b)

Fig.2 Schematic diagram of vehicle forces on different scenarios; (a) uphill; (b) downhill

To date, the existing studies on traffic flow assume that the current vehicle can accurately receive the traffic information of preceding vehicle. However, as discussed previously, the driving field of vision will be still limited in a traffic environment with gradient roads even though the $\mathrm{V} 2 \mathrm{~V}$ technology is in use, such that the sensors are difficult to accurately perceive the speed of front vehicle. In other words, certain errors of perceived speed may exist between the vehicle ahead and the real data. As a result, there is an imminent need to incorporate the velocity uncertainty of preceding vehicles in the design of car following control under such complex road environment. In view of this, we introduce the perception error term of preceding vehicle on Eq. (7), yielding a new traffic flow model as follows:

$$
\frac{d v_{n}(t)}{d t}=a\left[V^{o p}\left(y_{n}(t)\right)-v_{n}(t)\right]+a \lambda\left[(1+\varepsilon) v_{n+1}(t)-v_{n}(t)\right]+u_{n}(t)
$$

where $u_{n}(t)$ is the designed control input. $\varepsilon$ is the error coefficient representing the difference between the perceived data and real data; if $\varepsilon>0$, then the error is positive; otherwise, the error is negative; here we assume that $\|\varepsilon\|<1$. When $\varepsilon=0$, the proposed model is consistent with the literature [69]. Therefore, proposed new traffic flow model can be regarded as a generalized form of previous research.

\section{Control scheme for the car-following model}

In order to improve road traffic efficiency, we propose a new self-delayed feedback control for the car-following model. This control scheme only relies on the information of velocity and headway difference between the current time and historical time, specifically

$$
u_{j}(t)=k_{1}\left(y_{j}(t)-y_{j}\left(t-\tau_{1}\right)\right)+k_{2}\left(v_{j}(t)-v_{j}\left(t-\tau_{1}\right)\right)
$$

where $\tau_{1}$ is delay time. $k_{1}$ and $k_{2}$ are the weight coefficients corresponding to the headway and the speed difference, respectively.

Remark 1: The traditional linear feedback controller, with the vehicle speed difference or vehicle spacing difference of successive vehicles as the control input, requires accurate road traffic information. In the current underdeveloped traffic perception environment, the speed accuracy of preceding vehicle may be limited, such that the designed controller based on such information will greatly affect the control performance. On the contrary, the self-feedback controller Eq. (14) only relies on the speed and headway difference between the current time and the previous historical time, without additional external traffic information. As such, it can handle the issue of velocity uncertainty effect. In other words, the proposed controller Eq. (14) is particularly suitable for the 
current underdeveloped Internet of Vehicles environment.

To obtain the range of control gain coefficients $k_{1}$ and $k_{2}$, we perform stability analysis on the closed-loop traffic flow model under the feedback controller (14) based on the traditional control theory. Firstly, the steady state of the model is

$$
\left[y_{j}, v_{j}\right]^{T}=\left[y^{*}, v^{*}\right]^{T}
$$

where $y^{*}=h, v^{*}=\frac{V^{o p}(h)}{1-\lambda \varepsilon}$.

Let $\delta v_{j}=v_{j}-v^{*}$ and $\delta y_{j}=y_{j}-y^{*}$, substituting the above items into Eq. (13) and linearizing this equation yields the following equations:

$$
\left\{\begin{array}{l}
\frac{d \delta v_{n}(t)}{d t}=a\left[\Lambda \delta y_{n}(t)-\delta v_{n}(t)\right]+a \lambda\left[(1+\varepsilon) \delta v_{n+1}(t)-\delta v_{n}(t)\right]+ \\
k_{1}\left(\delta y_{j}(t)-\delta y_{j}\left(t-\tau_{1}\right)\right)+k_{2}\left(\delta v_{j}(t)-\delta v_{j}\left(t-\tau_{1}\right)\right) \\
\frac{d \delta y_{n}(t)}{d t}=\delta v_{n+1}(t)-\delta v_{n}(t)
\end{array}\right.
$$

where

$$
\Lambda=\left(\frac{v_{\max }-\sin \theta}{2}\right) V_{0}^{\prime} \quad, \quad V_{0}^{\prime}=\left.\frac{d V_{0}(\rho)}{d \rho}\right|_{\rho=\rho_{0}} \quad, \quad \delta y_{j+1}\left(t-\tau_{1}\right)=y_{j+1}\left(t-\tau_{1}\right)-y_{j}^{*} \quad,
$$

$\delta v_{j+1}\left(t-\tau_{1}\right)=v_{j+1}\left(t-\tau_{1}\right)-v_{j}^{*}$.

Applying the Laplace transform to the above formula, we have that

$$
\left\{\begin{array}{l}
s V_{n}(s)-V_{n}(0)=a\left[\Lambda Y_{n}(s)-V_{n}(s)\right]+a \lambda\left[(1+\varepsilon) V_{n+1}(s)-V_{n}(s)\right]+ \\
k_{1}\left(1-e^{-s \tau_{1}}\right) Y_{n}(s)+k_{2}\left(1-e^{-s \tau_{1}}\right) V_{n}(s) \\
s Y_{n}(s)-Y_{n}(0)=V_{n+1}(s)-V_{n}(s)
\end{array}\right.
$$

where $P_{j}(s)=L\left(\rho_{j}\right)$ and $Q_{j}(s)=L\left(q_{j}\right) . L(\mathrm{~g})$ represents the Laplace transform. $s$ is the complex variable.

By transforming Eq. (17) to eliminate the intermediate variable $Y_{n}(s)$, then the transition function of is

$$
G(s)=\frac{a \Lambda+a \lambda(1+\varepsilon) s+k_{1}\left(1-e^{-s \tau_{1}}\right)}{d(s)}
$$

where $d(s)$ represents the characteristic polynomial, and

$$
d(s)=s^{2}+a(1+\lambda) s+a \Lambda+k_{1}\left(1-e^{-s \tau_{1}}\right)-k_{2}\left(1-e^{-s \tau_{1}}\right) s .
$$

Lemma 1 ${ }^{[52]}$ : According to the classical control theory, the traffic flow is stable and the traffic jam phenomenon will disappear if the following conditions are established simultaneously:

I) $\quad d(s)$ is stable;

II) $\|G(s)\|_{\infty} \leq 1$;

For condition (I), to sort out the characteristic polynomial $d(s)$ we have

$$
d(s) \equiv P(s)+Q(s) e^{-s \tau_{1}}
$$

where $P(s)=s^{2}+\left(a+a \lambda-k_{2}\right) s+a \Lambda+k_{1}, Q(s)=k_{2} s-k_{1}$.

Furthermore, the formula (19) is written as follows: 


$$
\begin{aligned}
& F(w)=[\operatorname{Re} P(i w)]^{2}+[\operatorname{Im} P(i w)]^{2}-[\operatorname{Re} Q(i w)]^{2}-[\operatorname{Im} Q(i w)]^{2} \\
& =\left(-w^{2}+a \Lambda+k_{1}\right)^{2}+\left(a+a \lambda-k_{2}\right)^{2} w^{2}-k_{1}^{2}-k_{2}^{2} w^{2} \\
& =w^{4}+m_{1} w^{2}+m_{2}
\end{aligned}
$$

where $m_{1}=(a+a \lambda)^{2}-2 a(1+\lambda) k_{2}-2\left(a \Lambda+k_{1}\right), m_{2}=a^{2} \Lambda^{2}+2 a \Lambda k_{1}$.

Based on the literature [1], if the following conditions are met, then the critical function $F(w)$ has no real roots, specifically

$$
m_{1} \geq 0, m_{2} \geq 0 \text { or } m_{1}<0, m_{1}^{2}-4 m_{2}<0 .
$$

Substituting $m_{1}$ and $m_{2}$ into the formula (21), we have that

$$
a(1+\lambda) k_{2}+k_{1} \leq \frac{(a+a \lambda)^{2}}{2}-a\left(\frac{v_{\max }-\sin \theta}{2}\right) V_{0}^{\prime}, \quad k_{1} \geq \frac{-a\left(v_{\max }-\sin \theta\right) V_{0}^{\prime}}{4}
$$

or

$$
\begin{gathered}
a(1+\lambda) k_{2}+k_{1}>\frac{(a+a \lambda)^{2}}{2}-a\left(\frac{v_{\max }-\sin \theta}{2}\right) V_{0}^{\prime} \\
{\left[(a+a \lambda)^{2}-2 a(1+\lambda) k_{2}\right]^{2}+4 k_{1}^{2}<4\left[(a+a \lambda)^{2}-2 a(1+\lambda) k_{2}\right]\left[a\left(\frac{v_{\max }-\sin \theta}{2}\right) V_{0}^{\prime}+k_{1}\right]}
\end{gathered}
$$

In summary, if condition (22) or (23) is hold, then the characteristic polynomial $d(s)$ satisfy stable. Figs. 3 and 4 show the delay-independent stable region of $d(s)$ in phase diagram $\left(k_{1}, k_{2}\right)$ under downhill and uphill scenarios. The shaded region represents the set of parameters combination where characteristic polynomial $d(s)$ is guaranteed stable.

For condition (II), we have that

$$
\left\{\begin{aligned}
\|G(s)\|_{\infty} & =\sup _{w \in[0, \infty)}|G(i w)| \leq 1 \\
|G(i w)| & =\sqrt{G(i w) G(-i w)} \\
& =\sqrt{\frac{\left[a \Lambda+k_{1}\left(1-\cos \left(w \tau_{1}\right)\right)\right]^{2}+\left[a \lambda(1+\varepsilon) w+k_{1} \sin \left(w \tau_{1}\right)\right]^{2}}{\left[-w^{2}+a \Lambda+k_{1}\left(1-\cos \left(w \tau_{1}\right)\right)+k_{2} w \sin \left(w \tau_{1}\right)\right]^{2}+\left[a(1+\lambda) w+k_{1} \sin \left(w \tau_{1}\right)-k_{2} w\left(1-\cos \left(w \tau_{1}\right)\right)\right]^{2}}} \leq 1
\end{aligned}\right.
$$

Therefore, if the inequality $|G(i w)| \leq 1$ is hold for any $w \in[0,+\infty)$, then the following condition is met, specifically,

$$
\begin{aligned}
& {\left[-w^{2}+a \Lambda+k_{1}\left(1-\cos \left(w \tau_{1}\right)\right)+k_{2} w \sin \left(w \tau_{1}\right)\right]^{2}+\left[a(1+\lambda) w+k_{1} \sin \left(w \tau_{1}\right)-k_{2} w\left(1-\cos \left(w \tau_{1}\right)\right)\right]^{2}-} \\
& {\left[a \Lambda+k_{1}\left(1-\cos \left(w \tau_{1}\right)\right)\right]^{2}-\left[a \lambda(1+\varepsilon) w+k_{1} \sin \left(w \tau_{1}\right)\right]^{2} \geq 0}
\end{aligned}
$$

Theorem 1: Based on the traffic flow model (13), for given parameters $\lambda, k_{1}, k_{2}$ and $\tau_{1}$, if the conditions (22) (or (23)) and (25) are met, then traffic congestion can be effectively suppressed under the delay feedback controller (14).

Figs. 5 and 6 present the amplitude change of the transfer function under different key parameters $k_{1}, k_{2}$ and $\tau_{1}$ for the designed controller (14), where Fig. 5 and Fig. 6 correspond to downhill and uphill scenario, respectively. As shown in Fig. 5(a), the amplitude of the transfer function $|G|$ is effectively reduced as the parameters $k_{1}, k_{2}$ increase; when $k_{1}=2.5$ and $k_{2}=2.5$, the theorem 1 is satisfied, the amplitude vertex of the transition function is less than 1 . Therefore, the 
designed controller (14) is conducive to enhance the robustness of traffic flow. Fig. 5(b) compares the amplitude of the transfer function $|G|$ under different delay time $\tau_{1}$. When $\tau_{1}=0.5$, the amplitude of the bode curve is greater than 0 , and the traffic disturbance will exacerbate over time and induce traffic jam phenomenon. As the parameter $\tau_{1}$ continues to increase, the amplitude of bode curve keep decreases. When $\tau_{1}=2.5$, the amplitude of the bode curve is approximately less than 1 , which means that the traffic flow is stable. Therefore, it can be concluded that a higher value of parameter $\tau_{1}$ is conducive to improving the robustness of traffic flow.

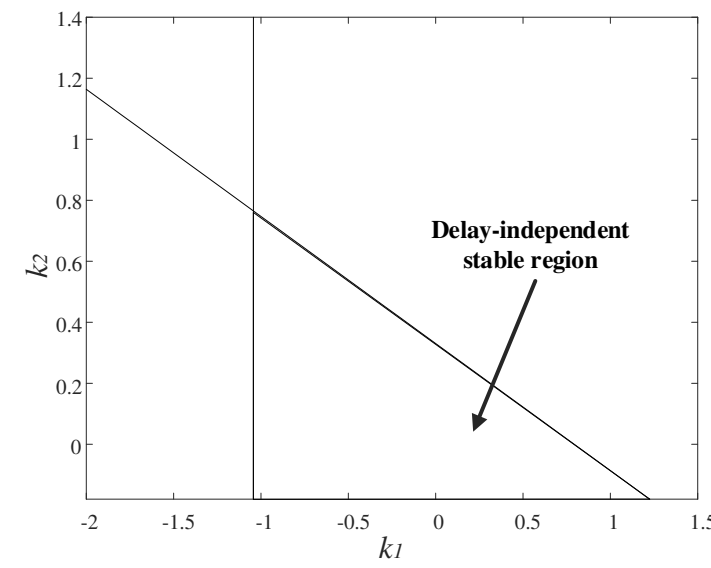

(a)

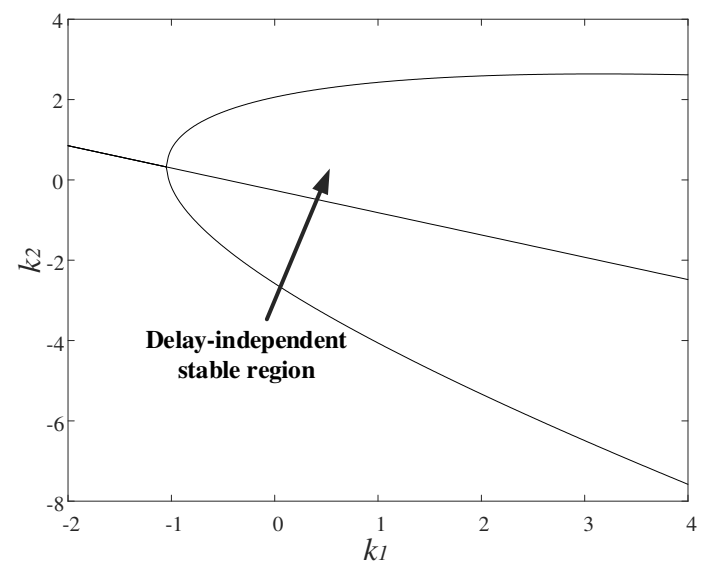

(b)

Fig.3 Delay-independent stable region of characteristic polynomial $d(s)$ on phase diagram $\left(k_{1}, k_{2}\right)$ with $a=2$, $\theta=-5^{\circ}, \quad \lambda=-0.1$; (a) condition (22); (b) condition (23).

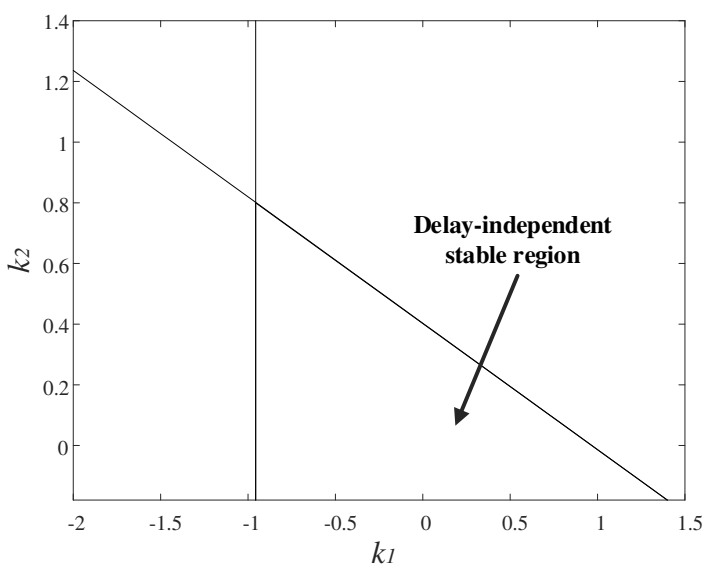

(a)

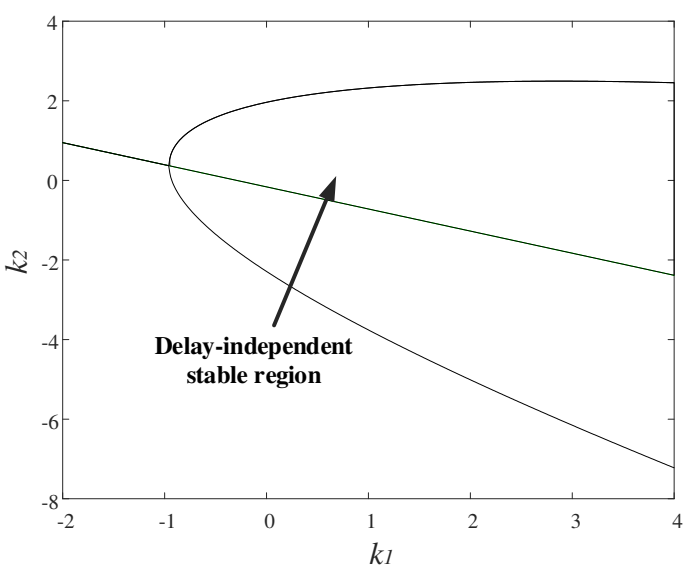

(b)

Fig.4 Delay-independent stable region of characteristic polynomial $d(s)$ on phase diagram $\left(k_{1}, k_{2}\right)$ with $a=2$, $\theta=5^{\circ}, \lambda=-0.1$; (a) condition (22); (b) condition (23). 


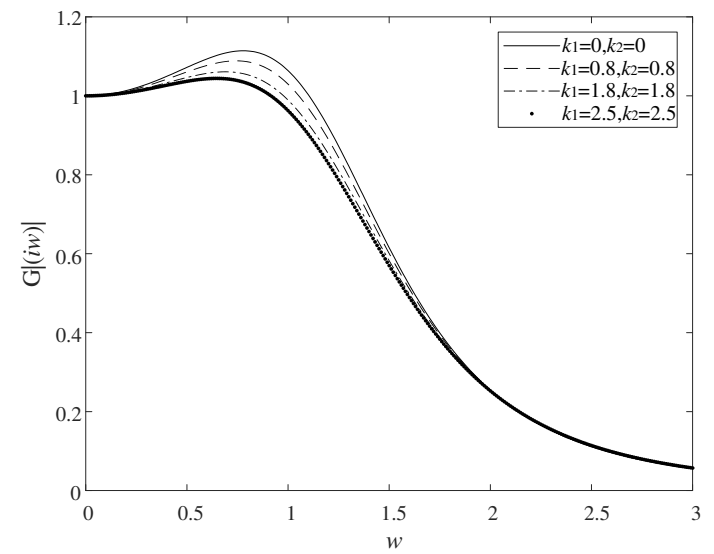

(a)

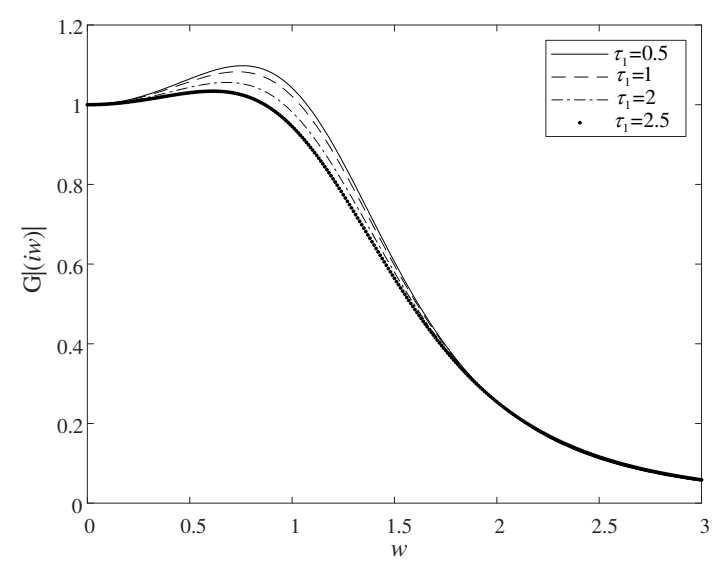

(b)

Fig.5. Bode-plot for different parameters (a) $k_{1}, k_{2}$, (b) $\tau_{1} \quad\left(\theta=-6^{o}\right)$

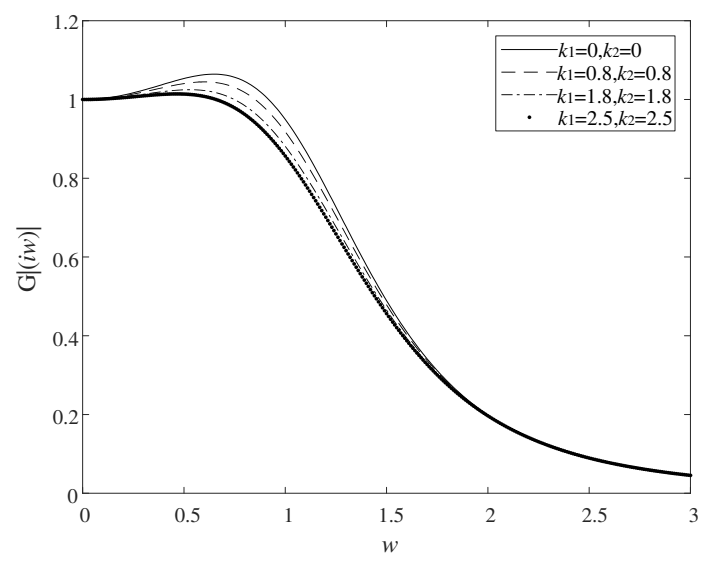

(a)

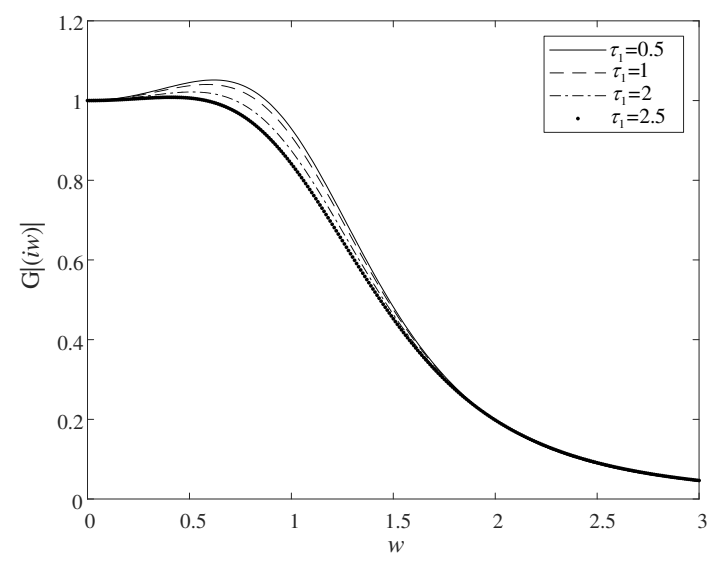

(b)

Fig.6. Bode-plot for different parameters (a) $k_{1}, k_{2}$, (b) $\tau_{1} \quad\left(\theta=6^{o}\right)$

\section{Simulation example}

In this section, we will perform numerical simulations under periodic boundary conditions to verify the effect of designed self-delay feedback controller on suppressing traffic congestion and reducing energy consumption. The initial position information is selected as follows:

$$
x_{n}(0)=\left\{\begin{array}{l}
4, n \neq \frac{N}{2}, \frac{N}{2}+1 \\
4-\delta, n=\frac{N}{2} \\
4+\delta, n=\frac{N}{2}+1
\end{array}\right.
$$

where the number of vehicles $N=100, \delta$ is the disturbance term, i.e., $\delta=0.1$, the other default parameters are selected as follows:

$$
v_{\max }=2, y_{c}=4, a=1.2, \lambda=-0.1, \varepsilon=0.1 .
$$

Fig. 7 shows the spatiotemporal evolution of the headways with different controller gains coefficient $k_{1}, k_{2}$ under the downhill scenario. Fig. 7(a) corresponds to the traffic flow without designed controller $\left(k_{1}, k_{2}=0\right)$. It shows that the headways on the road oscillate frequently with stop-and-go waves, which indicates that the traffic jam prone to occur. With the increasing of control gain coefficients $k_{1}, k_{2}$, the fluctuating frequency and amplitude of density waves have been effectively reduced. Typically, when $k_{1}=2, k_{2}=2$, the initial disturbance has been completely suppressed, and the stop-and-go phenomenon disappears. Therefore, higher values of parameter $k_{1}$, 
$k_{2}$ contributes to suppressing traffic congestion.

Fig. 8 shows the instantaneous headway distribution of all vehicles corresponding to Fig.7 at $t=10300$ s. As we can see, the fluctuating amplitude of headways without control in Fig.8 (a) is the largest, while the fluctuating amplitude is approximately equal to 0 in Fig. 14(d) indicating that the traffic flow is stable.

Fig. 9 describes the evolution of the headway between vehicles on the road under different values of parameter $\tau_{1}$. One can see that the headway fluctuation gradually decreases over time with the increase of parameter $\tau_{1}$, which suggests that a higher value of parameter $\tau_{1}$ is conducive to improving the anti-interference ability of traffic flow.

Fig. 10 shows the instantaneous headway distribution of all vehicles corresponding to Fig.9 at $t=10300 \mathrm{~s}$. We can see that the fluctuating amplitude of headways has been effectively reduced with the increase of parameter $\tau_{1}$, and the fluctuating amplitude between vehicles can be neglected in Fig.10(d). In other words, the initial disturbance has been completely diluted when the designed controller (14) is applied.

Figs.11-14 analyze the relationship between control gain coefficients $k_{1}, k_{2}$, delay time $\tau_{1}$ and traffic flow stability for the uphill scenario. With the increase of the gain coefficients $k_{1}, k_{2}$ or delay time, $\tau_{1}$, the robustness of traffic flow model can be enhanced. Therefore, the designed self-delayed feedback controller is effective in suppressing traffic congestion and enhancing the anti-interference ability for both the uphill or downhill scenarios.

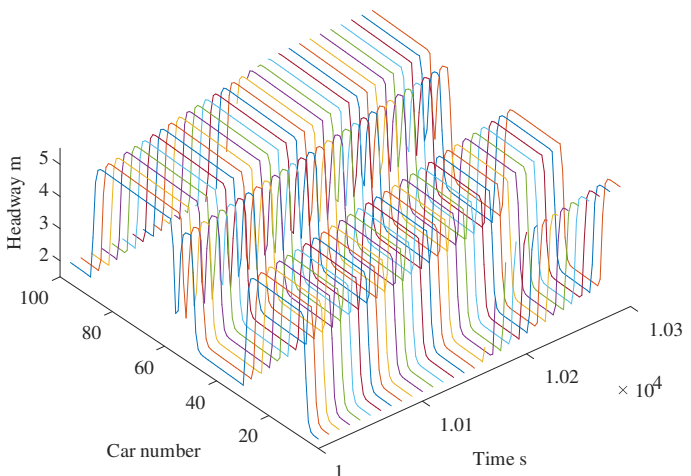

(a)

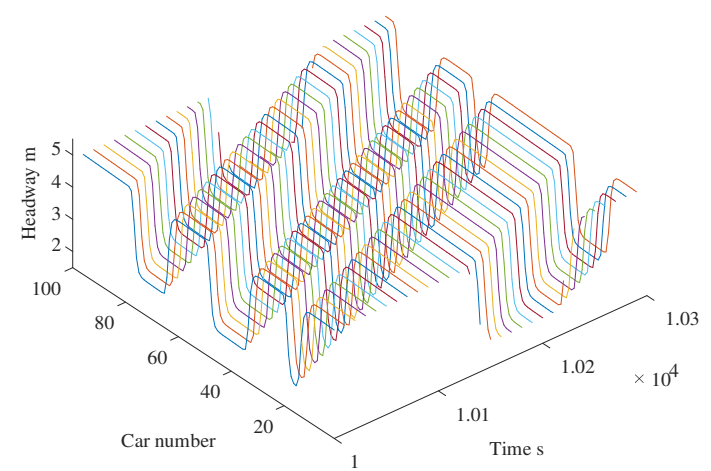

(c)

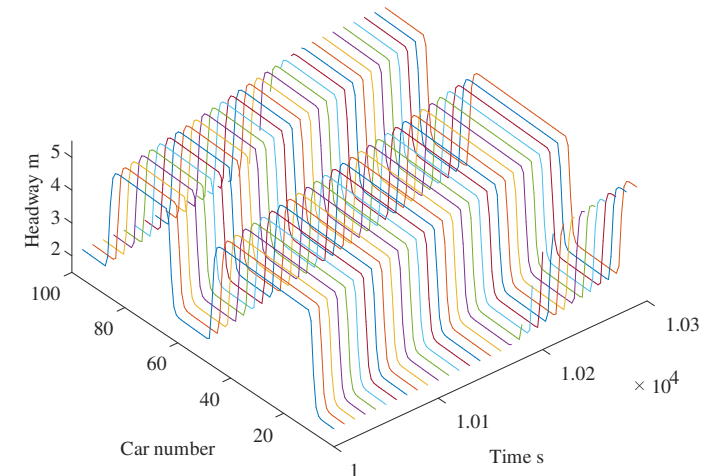

(b)

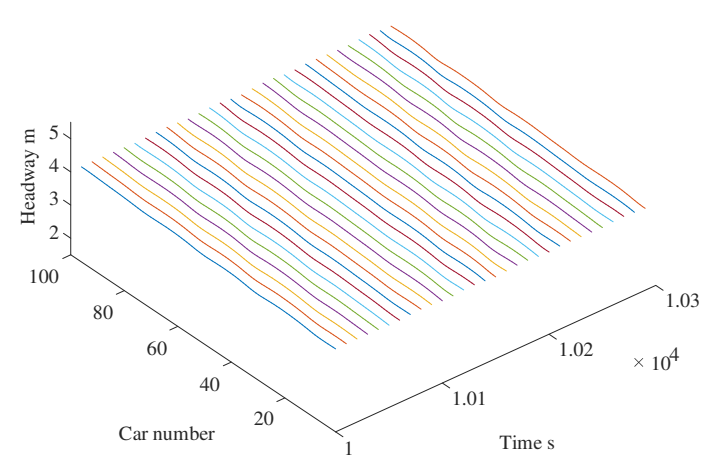

(d)

Fig.7. The spatio-temporal evolutions of headways under different values of parameter $k_{1}$ and $k_{2}$ after $t=10^{4} \mathrm{~s}$, where (a) $k_{1}=0, k_{2}=0$; (b) $k_{1}=0.5, k_{2}=0.5$;(c) $k_{1}=1, k_{2}=1$; (d) $k_{1}=2, k_{2}=2 .\left(\tau_{1}=0.1, \theta=-6^{o}\right)$ 


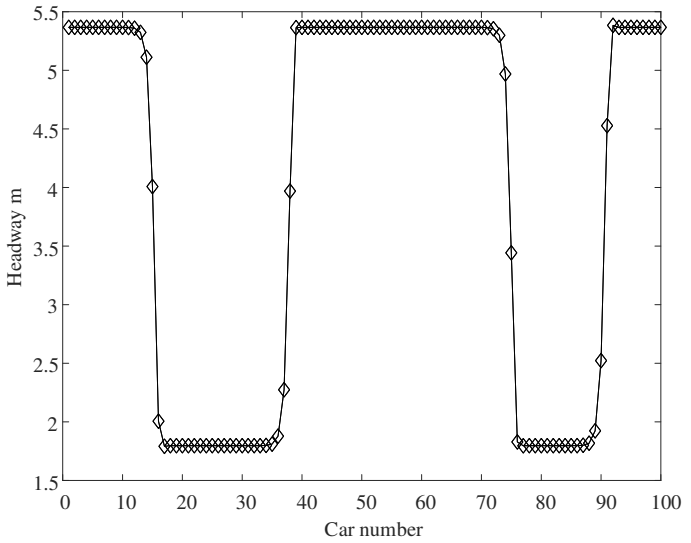

(a)

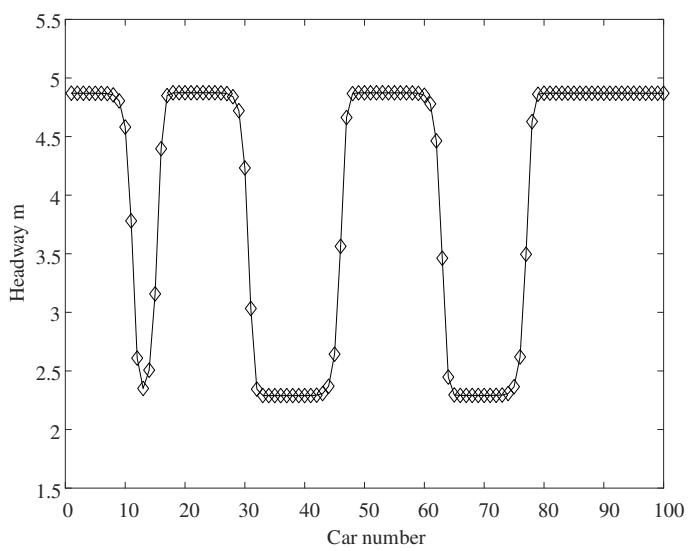

(c)

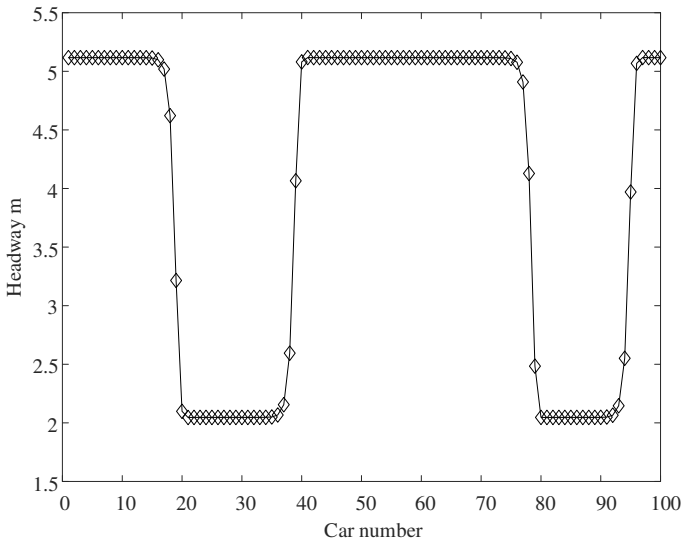

(b)

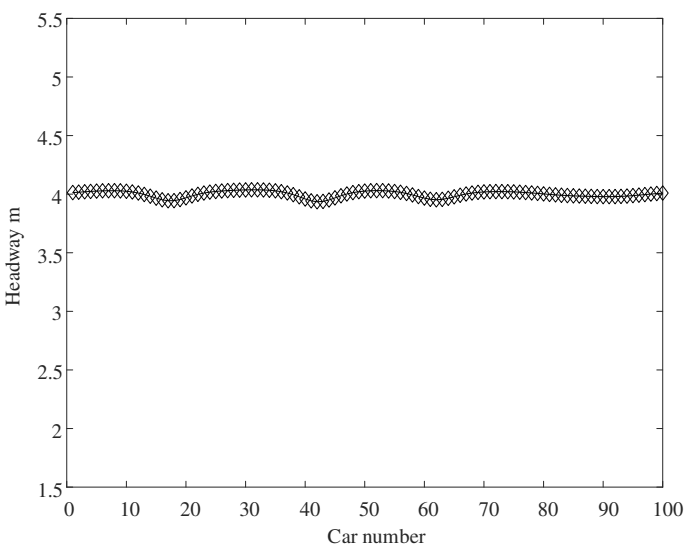

(d)

Fig.8. The instantaneous headway distribution between vehicles along the road corresponding to Fig.7

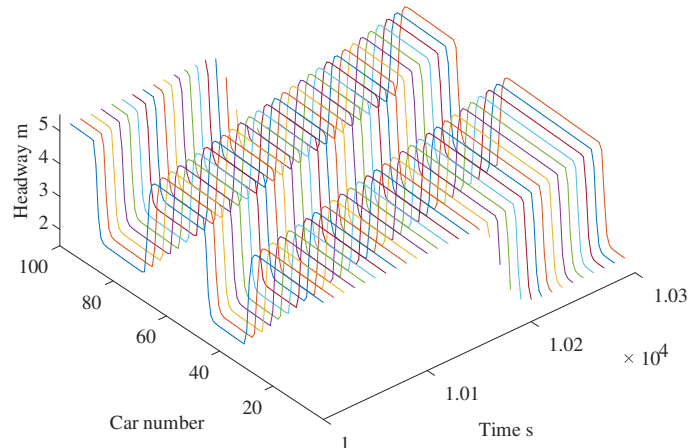

(a)

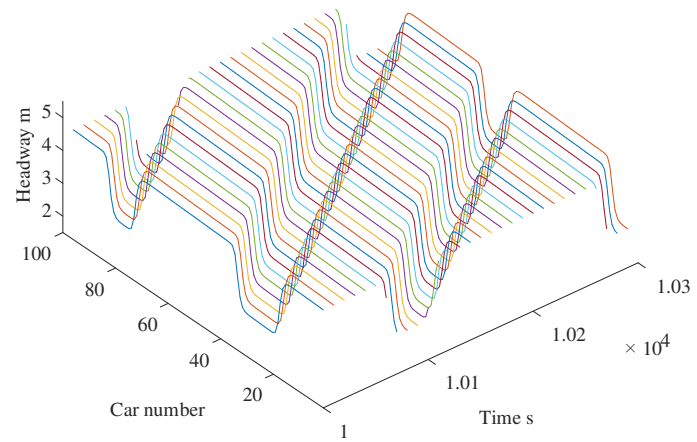

(c)

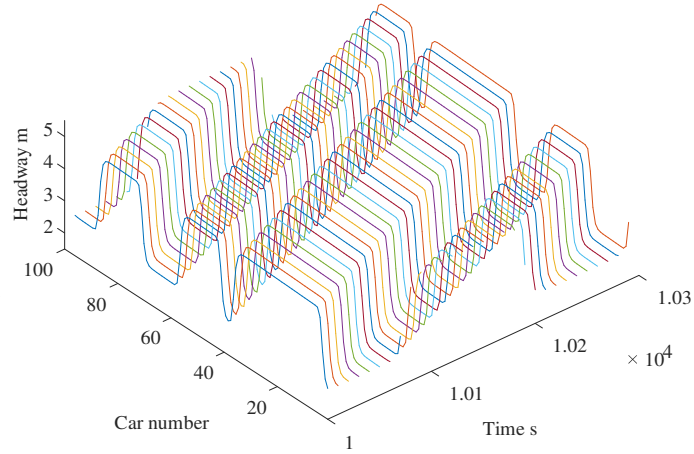

(b)

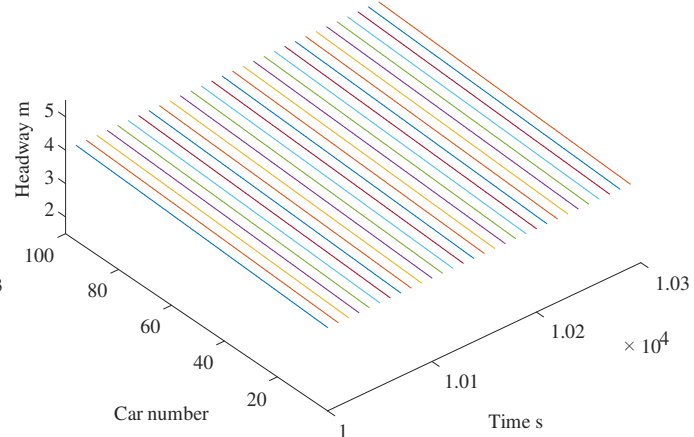

(d)

Fig.9. The spatio-temporal evolutions of headway under different values of parameter $\tau_{1}$ after $t=10^{4} \mathrm{~s}$, where (a) 

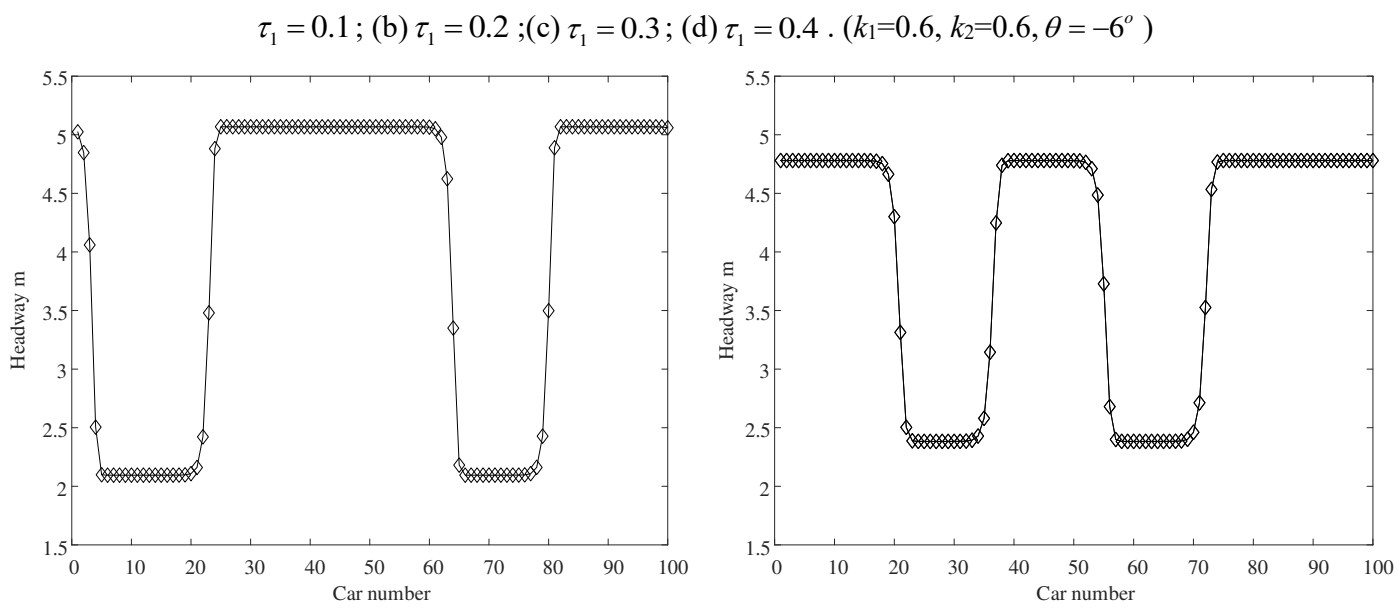

(a)

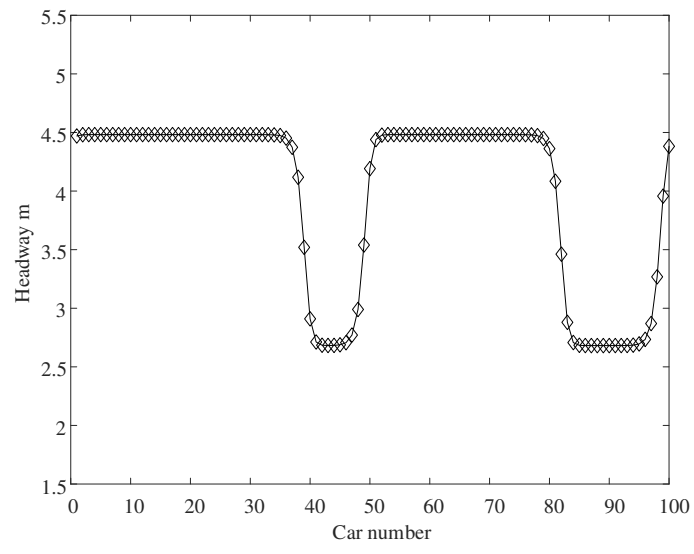

(b)

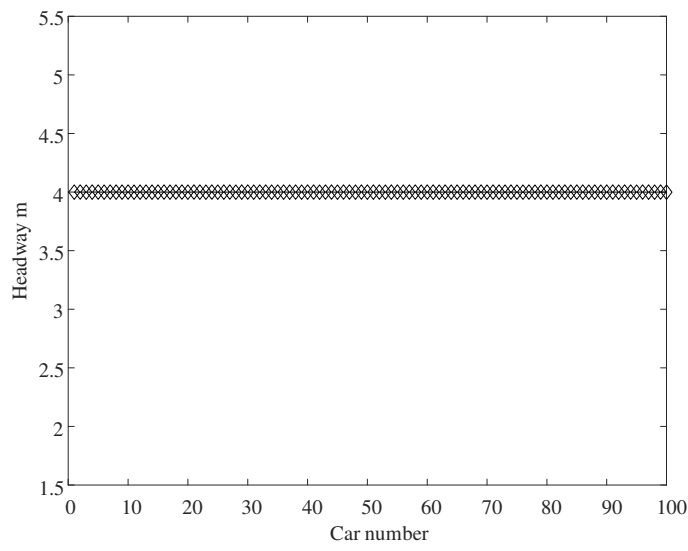

(d)

Fig.10. The instantaneous headway distribution between vehicles along the road corresponding to Fig.9

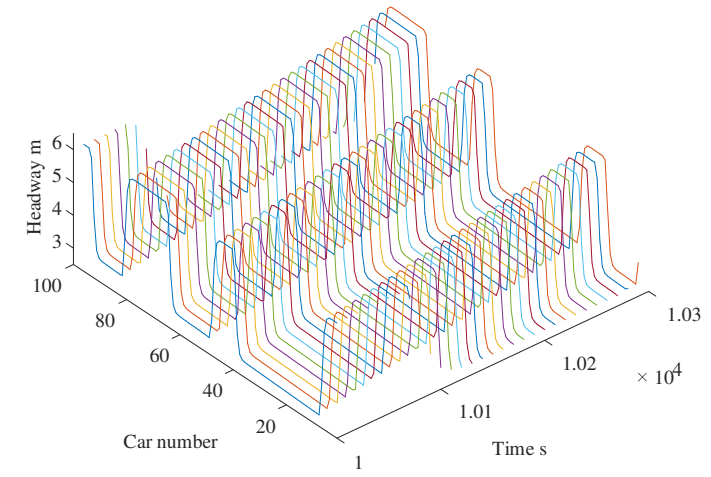

(a)

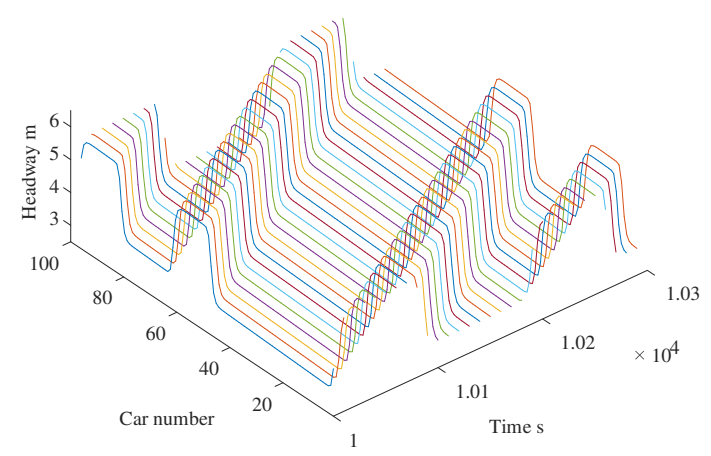

(c)

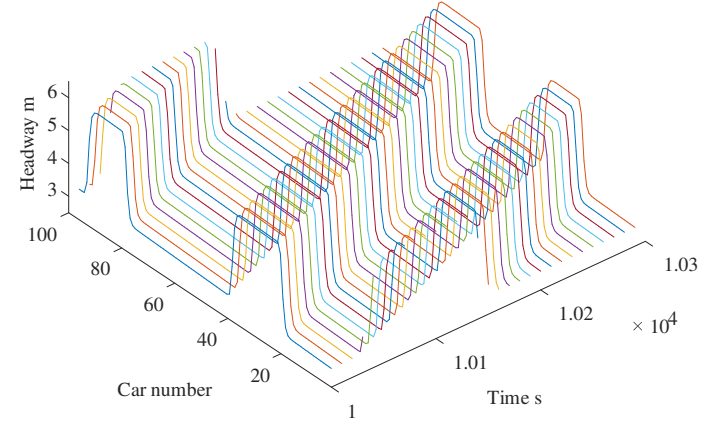

(b)

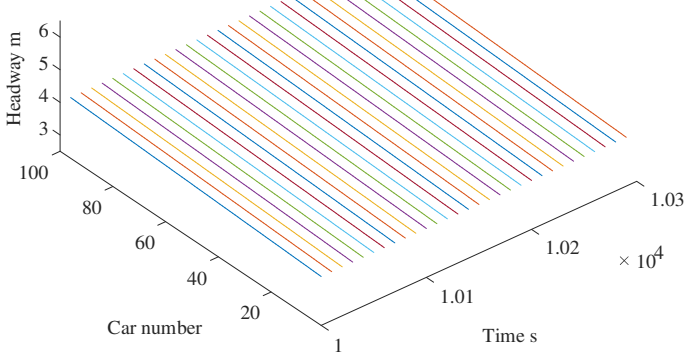

(d) 
Fig.11. The spatio-temporal evolutions of headway under different values of parameter $k_{1}$ and $k_{2}$ after $t=10^{4} \mathrm{~s}$, where (a) $k_{1}=0, k_{2}=0$; (b) $k_{1}=0.5, k_{2}=0.5$;(c) $k_{1}=1, k_{2}=1$; (d) $k_{1}=2, k_{2}=2 .\left(\tau_{1}=0.1, \theta=6^{\circ}\right)$

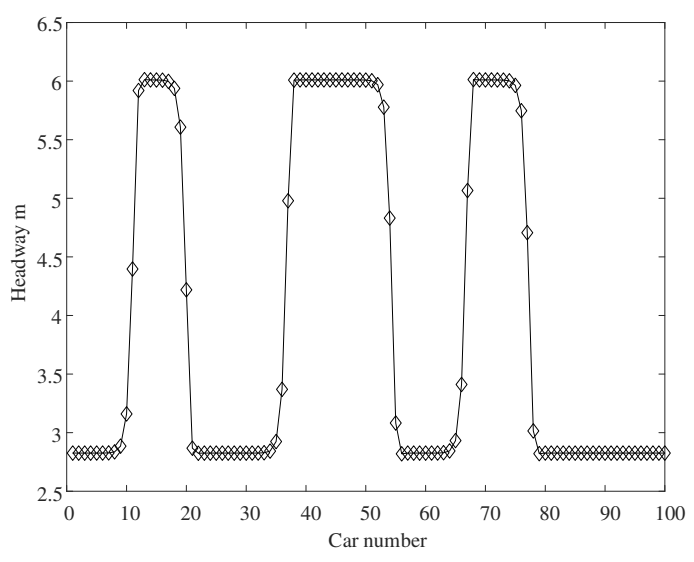

(a)

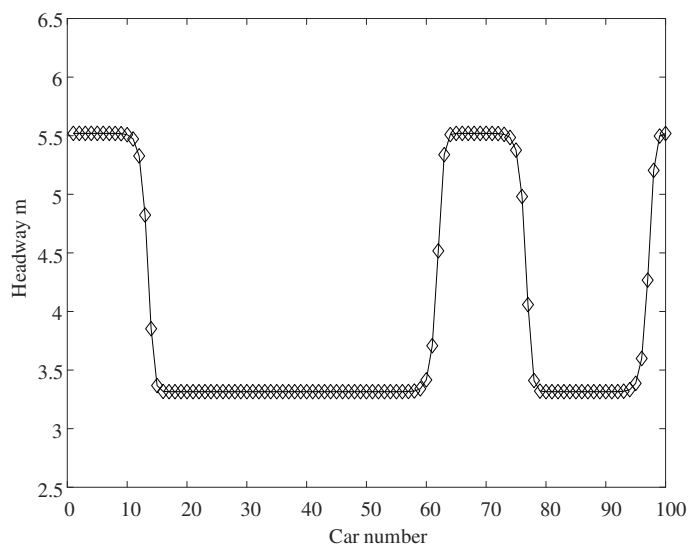

(c)

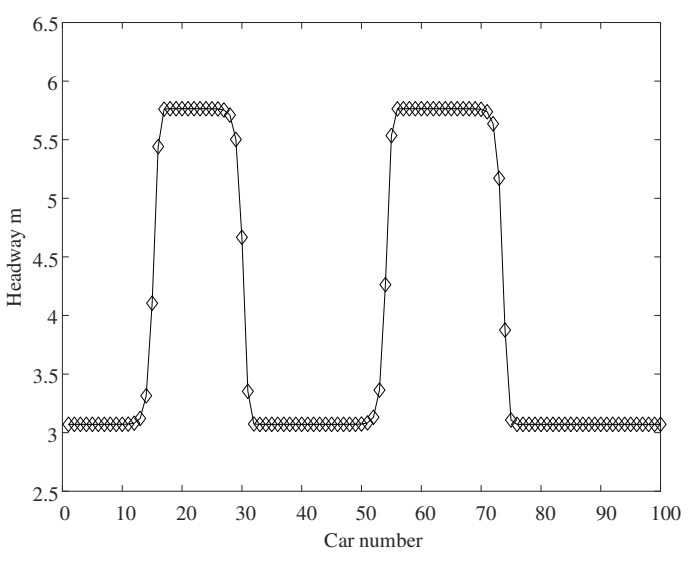

(b)

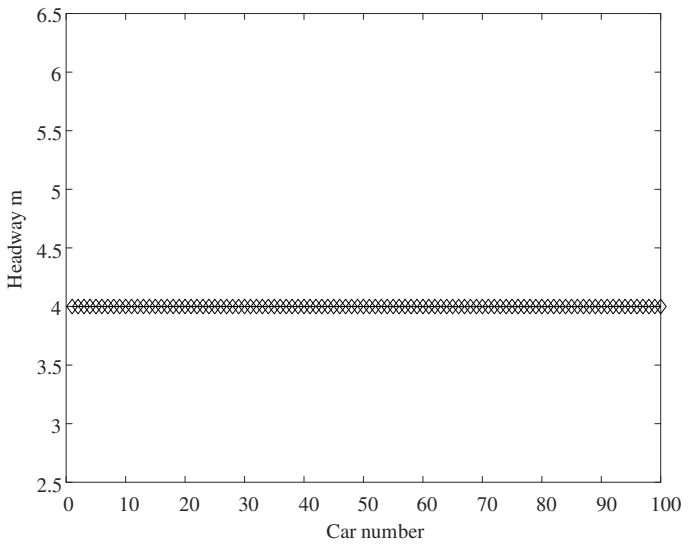

(d)

Fig.12. The instantaneous headway distribution between vehicles along the road corresponding to Fig.11

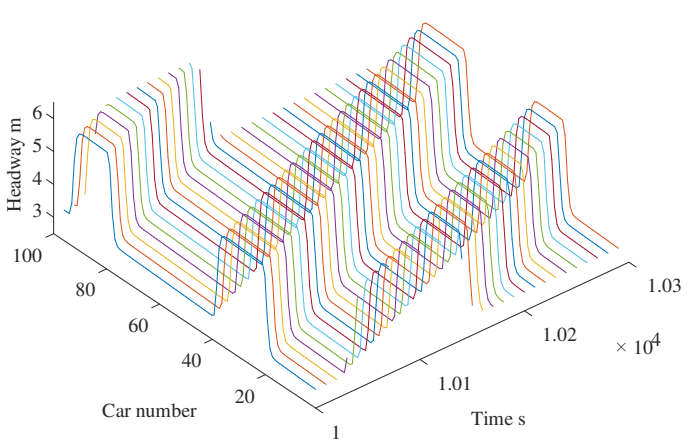

(a)

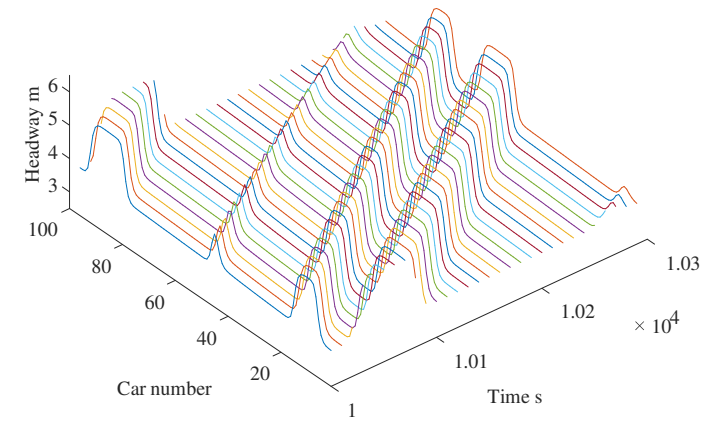

(c)

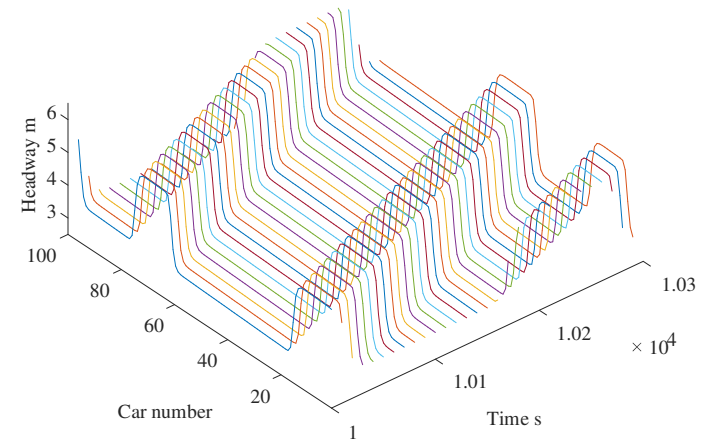

(b)

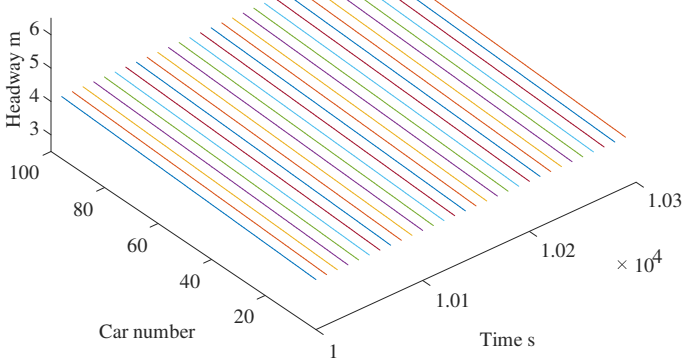

(d) 
Fig.13. The spatio-temporal evolutions of headway under different values of parameter $\tau_{1}$ after $t=10^{4} \mathrm{~s}$, where (a)

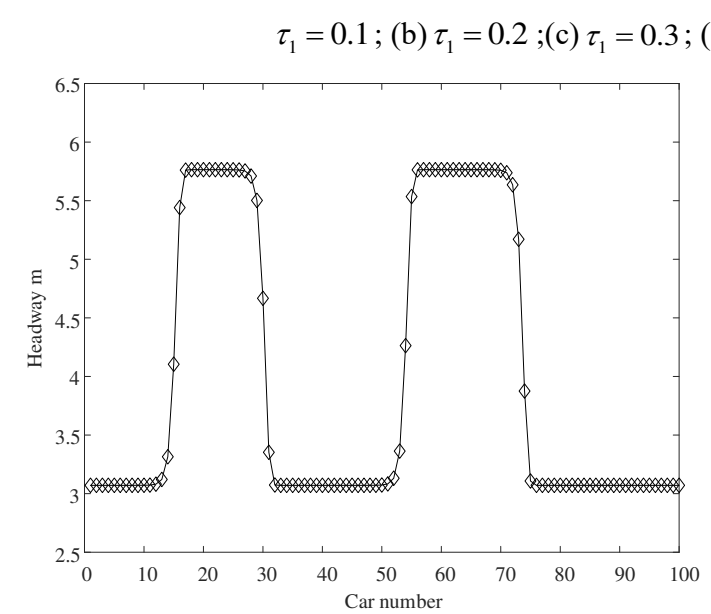

(a)

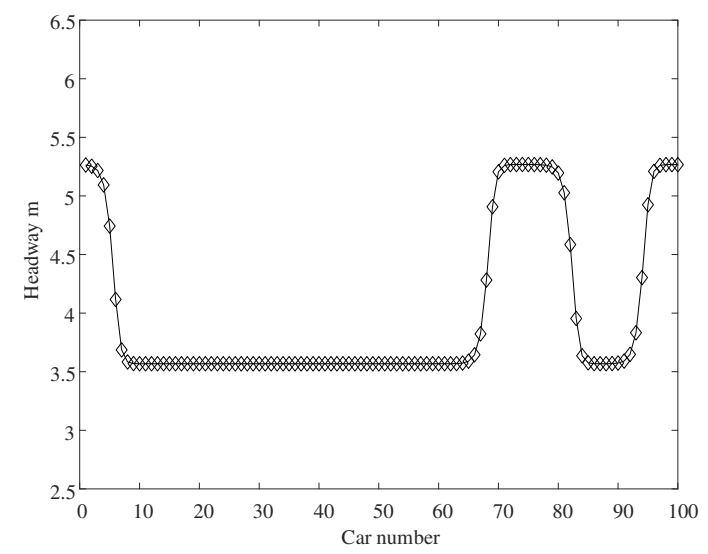

(c)

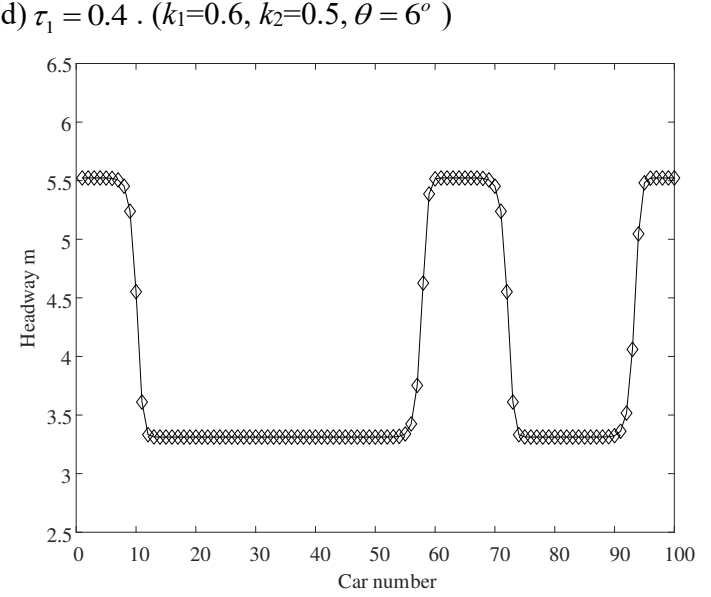

(b)

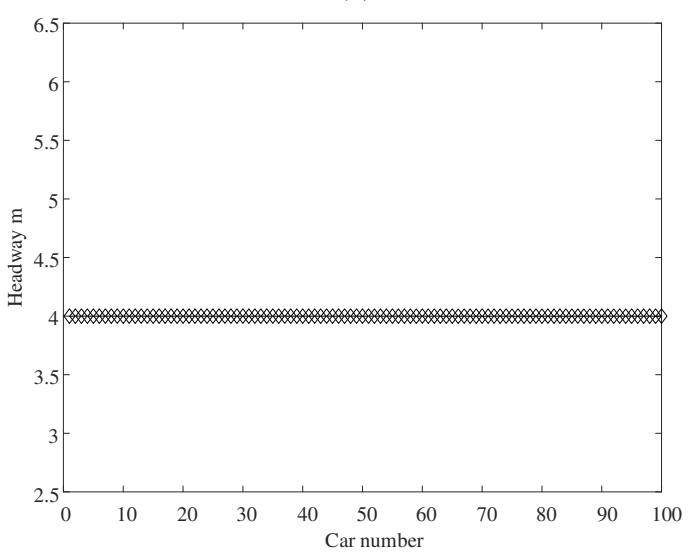

(d)

Fig.14. The instantaneous headway distribution corresponding to Fig. 13

\section{Conclusion}

This paper proposes a new self-delayed feedback controller for the car-following model with velocity uncertainty of preceding vehicle on gradient road. The stability condition of closed-loop traffic flow model is obtained via Hurwitz criteria and $H_{\infty}$ norm of transfer functions. We investigate the influence of parameters $k_{1}, k_{2}$ and $\tau_{1}$ on traffic jam from the uphill and downhill scenarios. Results show that as parameters of $k_{1}, k_{2}$ increases, the traffic congestion can be effectively suppressed. A higher value of delay time $\tau_{1}$ will decrease the fluctuation amplitude of headway, which indicates that the robustness of the traffic flow model is enhanced.

Despite the newly developed autonomous traffic flow model, there are a few strong assumptions in the current research. For example, the delay time term in designed feedback controller is constant, whereas the parameter uncertainties may be more realistic in the complex traffic environment[73]. In addition, we only verify the feasibility of designed control scheme without using real traffic data. These issues will be addressed in the follow-up research.

\section{Acknowledgement}

This work is jointly supported by the Regional Joint Fund for foundation and Applied Research Fund of Guangdong Province (Project No. 2019A1515111200, 2019A1515110837), the Science and Technology Program of Guangdong Province (Project No. 2020A1414010010), and 
the National Science Foundation of China (Project No. 72071079).

\section{Reference}

[1] Wu W, Liu R, Jin W, et al. Stochastic bus schedule coordination considering demand assignment and rerouting of passengers. Transportation Research Part B, 2019, 121:275-303.

[2] Wu W, Li P, Liu R, et al. Predicting peak load of bus routes with supply optimization and scaled Shepard interpolation: A newsvendor model. Transportation Research Part E, 2020, 142, 102041.

[3] Wu W, Lin Y, Liu R, et al. Online EV charge scheduling based on time-of-use pricing and peak load minimization: Properties and efficient algorithms. IEEE Transactions on Intelligent Transportation Systems, 2020, doi: 10.1109/TITS.2020.3014088.

[4] Zhang Y, Zhao M, Sun D, et al. An extended continuum mixed traffic model. Nonlinear Dynamics, 2021,103:1981-1909.

[5] Zhai C, Wu W. A continuum model with traffic interruption probability and electronic throttle opening angle effect under connected vehicle environment. The European Physical Journal B, 2020, 93, 52.

[6] Zhai C, Wu W. Analysis of driver's characteristics on continuum model with traffic jerk effect. Physics Letters A, 2018, 382(47): 3381-3392.

[7] Xue Y, Zhang Y, Fan D, et al. An extended macroscopic model for traffic flow on curved road and its numerical simulation. Nonlinear Dynamics, 2019, 95:3295-3307.

[8] Zhai C, Wu W. A new continuum model with driver's continuum sensory memory and preceding vehicle's taillight. Communications in Theoretical Physics, 2020,72(10):105004.

[9] Zhai C, Wu W. Lattice hydrodynamic modeling with continuous self-delayed traffic flux integral and vehicle overtaking effect. Modern Physics Letters B, 2020, 34(5): 2050071.

[10] Zhai $\mathrm{C}, \mathrm{Wu} \mathrm{W}$. A new lattice hydrodynamic model for bidirectional pedestrian flow with consideration of pedestrians' honk effect. International Journal of Modern Physics C, 2020, 31(2): 2050031.

[11] Zhai C, Wu W. Lattice hydrodynamic model based feedback control method with traffic interrupt probability. Modern Physics Letters B, 2019, 33(23): 1950273.

[12] Zhai $\mathrm{C}, \mathrm{Wu} \mathrm{W}$. An extended multi-phase lattice model with consideration of optimal current changes with memory. Cluster Computing, 2019, 22: 7447-7457.

[13] Jiao C, Cheng R, Ge H. An improved lattice hydrodynamic model considering the "backward looking" effect and the traffic interruption probability. Nonlinear Dynamics, 2018, 91:777-784.

[14] Zhai C, Wu W. Lattice hydrodynamic modeling with continuous self-delayed traffic flux integral and vehicle overtaking effect. Modern Physics Letters B, 2020, 34(5): 2050071.

[15] Zhai C, Wu W. Designing continuous delay feedback control for lattice hydrodynamic model under cyber-attacks and connected vehicle environment. Communications in Nonlinear Science and Numerical Simulation, 2021, 95:105667.

[16] Pipes L. An operational analysis of traffic dynamics. Journal of Applied Physics, 1953, 24(3):274-281.

[17] Newell G. Nonlinear effects in the dynamics of car following. Operations Research, 1961, 9(2):209-229.

[18] Gipps P. A behavioural car-following model for computer simulation. Transportation Research Part B: Methodological, 1981, 15(2):105-111.

[19] Zeng J, Qian Y, Mi P, et al. Freeway traffic flow cellular automata model based on mean velocity feedback. Physica A: Statistical Mechanics \& Its Applications, 2021, 562: 125387.

[20] Xue Y, Wang X, Cen B, et al. Study on fuel consumption in the Kerner-Klenov-Wolf three phase cellular automation traffic flow model. Nonlinear Dynamics, 2020, 102:393-402.

[21] Bando M, Hasebe K, Nakayama A, et al. Dynamical model of traffic congestion and numerical simulation. Physical Review E Statistical Physics Plasmas Fluids \& Related Interdisciplinary Topics, 1995, 51(2):1035-1042.

[22] Jiao S, Zhang S, Zhou B, et al. Dynamic performance and safety analysis of car following models considering collision sensitivity. Physica A: Statistical Mechanics \& Its Applications, 2021, 564: 125504.

[23] Yu S, Liu Q, Li X. Full velocity difference and acceleration model for a car following theory. Communications in Nonlinear Science and Numerical Simulation, 2013, 18:1229-1234.

[24] Zhao X, Gao Z. A new car following model: full velocity and acceleration difference model. The European Physical Journal B, 2005, 47, 145-150.

[25] Xu X, Pang J, Monterola C. Asymmetric optimal velocity car following model. Physica A: Statistical 
Mechanics \& Its Applications, 2015, 436: 565-571.

[26] Sun Y, Ge H, Cheng R. An extended car following model considering driver's memory and average speed of preceding vehicles with control strategy. Physica A: Statistical Mechanics \& Its Applications, 2019, 521: 752-761.

[27] Jin Z, Yang Z, Ge H. Energy consumption investigation for a new car following model considering driver's memory and average speed of the vehicles. Physica A: Statistical Mechanics \& Its Applications, 2018, 506: 1038-1049.

[28] Sun D, Kang Y, Yang S. A novel car following model considering average speed of preceding vehicles group. Physica A: Statistical Mechanics \& Its Applications, 2015, 436: 103-109.

[29] Ma G, Ma M, Liang S, et al. Nonlinear analysis of the car following model considering headway changes with memory and backward looking effect. Physica A: Statistical Mechanics \& Its Applications, 2021, 562: 125303.

[30] Ma G, Ma M, Liang S, et al. An improved car following model accounting for the time delayed velocity difference and backward looking effect. Communications in Nonlinear Science and Numerical Simulation, 2020 , 85: 105221

[31] Ma M, Ma G, Liang S. Density waves in car following model for autonomous vehicles with backward looking effect. Applied Mathematical Modelling, 2021, 94:1-12.

[32] Chen C, Cheng R, Ge H. An extended car following model considering driver's sensory memory and the backward looking effect. Physica A: Statistical Mechanics \& Its Applications, 2019, 525: 278-289.

[33] Li S, Cheng R, Ge H. An improved car following model considering electronic throttle dynamics and delayed velocity difference. Physica A: Statistical Mechanics \& Its Applications, 2020, 558: 125015.

[34] Sun Y, Ge H, Cheng R. A car following model considering the effect of electronic throttle opening angle over the curved road. Physica A: Statistical Mechanics \& Its Applications, 2019, 534: 122377.

[35] Li Y, Li Z, Peeta S, et al. A car following model considering the effect of electronic throttle opening angle under connected environment. Nonlinear Dynamics, 2016, 85:2115-2125.

[36] Yan C, Ge H, Cheng R. An extended car following model by considering the optimal velocity difference and electronic throttle angle. Physica A: Statistical Mechanics \& Its Applications, 2019, 535: 122216.

[37] Hou P, Yu H, Yan C, et al. An extended car following model based on visual angle and backward looking effect. Chinese Journal of Physics, 2017, 55:2092-2099.

[38] Zhou J. An extended visual angle model for car following theory. Nonlinear Dynamics, 2015, 81:549-560.

[39] Zheng L, Zhou S, Jin P, et al. Influence of lateral discomfort on the stability of traffic flow based on visual angle car following model. Physica A: Statistical Mechanics \& Its Applications, 2012, 391: 5948-5959.

[40] Li Y, Zhang L, Zheng H, et al. Evaluating the energy consumption of electric vehicles based on car following model under non-lane discipline. Nonlinear Dynamics, 2015, 82:629-641.

[41] Li Y, Zhang L, Peeta S, et al. Non-lane-discipline- based car following model considering the effects of two-sided lateral gaps. Nonlinear Dynamics, 2015, 80:227-238.

[42] Jin S, Wang D, Tao P, et al. Non-lane-based full velocity difference car following model. Physica A: Statistical Mechanics \& Its Applications, 2010, 389: 4654-4662.

[43] Li Y, Zhang L, Zhang B, et al. Non-lane-discipline-based car following model considering the effect of visual angle. Nonlinear Dynamics, 2016, 85:1901-1912.

[44] Tang T, Luo X, Liu K. Impacts of the driver's bounded rationality on the traffic running cost under the car following model. Physica A: Statistical Mechanics \& Its Applications, 2016, 457: 316-321.

[45] Tang T, Huang H, Shang H. Influences of the driver's bounded rationality on micro driving behavior, fuel consumption and emissions. Transportation Research Part D: Transport and Environment, 2015,41:423-432.

[46] Zhai C, Wu W. A new car-following model considering driver's characteristics and traffic jerk. Nonlinear Dynamics, 2018, 93: 2185-2199.

[47] Song $\mathrm{H}$, Ge $\mathrm{H}$, Chen $\mathrm{F}$, et al. TDGL and $\mathrm{mKdV}$ equations for car following model considering traffic jerk and velocity difference. Nonlinear Dynamics, 2017, 87: 1809-1817.

[48] Liu F, Cheng R, Zheng P, et al. TDGL and mKdV equations for car following model considering traffic jerk. Nonlinear Dynamics, 2016, 83: 793-800.

[49] Tang T, Huang H, Wong S, et al. A new car following model with considering the traffic interruption probability. Chinese Physics B, 2009, 18(3):975.

[50] Zhang G, Liu H. Effect of current vehicle's interruption on traffic stability in cooperative car following model. Modern Physics Letters B, 2017, 31(34): 1750317.

[51] Li Y, Sun D, Liu W, et al. Modeling and simulation for microscopic traffic flow based on multiple headway, velocity and acceleration difference. Nonlinear Dynamics, 2011, 66: 15-28. 
[52] Kuang H, Wang M, Liu F, et al. An extended car following model considering multi-anticipative average velocity effect under V2V environment. Physica A: Statistical Mechanics \& Its Applications, 2019, 527: 121268.

[53] Tang T, Li C, Wu Y, et al. Impact of the honk effect on the stability of traffic flow. Physica A: Statistical Mechanics \& Its Applications, 2011, 390: 3362-3368.

[54] Kuang H, Xu Z, Li X, et al. An extended car following model accounting for the honk effect and numerical tests. Nonlinear Dynamics, 2017, 87: 149-157.

[55] Zhai C, Wu W. Car-following model based delay feedback control method with the gyroidal road. International Journal of Modern Physics C, 2019, 30(9): 1950073.

[56] Sun Y, Ge H, Cheng R. An extended car following model considering driver's desire for smooth driving on the curved road. Physica A: Statistical Mechanics \& Its Applications, 2019, 527: 121426.

[57] Cao B. A new car following model considering driver's sensory memory. Physica A: Statistical Mechanics \& Its Applications, 2015, 427: 218-225.

[58] Yu S, Shi Z. An improved car following model considering headway changes with memory. Physica A: Statistical Mechanics \& Its Applications, 2015, 421: 1-14.

[59] Wang Y, Song H, Cheng R. TDGL and mKdV equations for an extended car following model with the consideration of driver's memory. Physica A: Statistical Mechanics \& Its Applications, 2019, 515: 440-449.

[60] Cheng J, Liu R, Ngoduy D, et al. A new multi-anticipative car following model with consideration of the desired following distance. Nonlinear Dynamics, 2016, 85: 2705-2717.

[61] Wang T, Li G, Zhang J, et al. The effect of headway variation tendency on traffic flow: modeling and stabilization. Physica A: Statistical Mechanics \& Its Applications, 2019, 525: 566-575.

[62] Zhou T, Sun D, Kang Y, et al. A new car following model with consideration of the prevision driving behavior. Communications in Nonlinear Science and Numerical Simulation, 2014, 19:3820-3826.

[63] Yu L, Shi Z, Li T. A new car following model with two delays. Physics Letters A, 2014, 378: 348-357.

[64] Li S, Yang L, Gao Z, et al. Stabilization strategies of a general nonlinear car following model with carrying reaction time delay of the drivers. ISA Transactions, 2014,53:1739-1745.

[65] Peng G, He H, Lu W. A new car following model with the consideration of incorporating timid and aggressive driving behaviors. Physica A: Statistical Mechanics \& Its Applications, 2016, 442: 197-202.

[66] Tang T, He J, Yang S, et al. A car following model accounting for the driver's attribution. Physica A: Statistical Mechanics \& Its Applications, 2014, 413: 583-591.

[67] Wang J, Sun F, Ge H. Effect of the driver's desire for smooth driving on the car following model. Physica A: Statistical Mechanics \& Its Applications, 2018, 512: 96-108.

[68] Sun Y, Ge H, Cheng R. An extended car following model considering driver's desire for smooth driving on the curved road. Physica A: Statistical Mechanics \& Its Applications, 2019, 527: 121426.

[69] Yang S, Li M, Tang T. Electronic vehicle's electricity consumption on a road with different slope. Physica A: Statistical Mechanics \& Its Applications, 2014, 402: 41-48.

[70] Kaur R, Sharma S. Modeling and simulation of driver's anticipation effect on a two lanes system on curved road with slope. Physica A: Statistical Mechanics \& Its Applications, 2018, 499: 110-120.

[71] Helbing D, Tilch B. Generalized force model of traffic dynamics. Physical Review E, 1998, 58(1): 133-138

[72] Jiang R, Wu Q, Zhu Z. Full velocity difference model for a car following theory. Physical Review E, 2001, 64(1): 017101.

[73] Chen D, Sun D, Liu H, et al. Robust control for cooperative driving system of heterogeneous vehicles with parameter uncertainties and communication constraints in the vicinity of traffic signals. Nonlinear Dynamics, 2020, 99: $1659-1674$. 